\title{
Interference-Based Decode and Forward Scheme Using Relay Nodes in Heterogeneous Networks
}

\author{
Kentaro Nishimori, ${ }^{1}$ Koshiro Kitao, ${ }^{2}$ and Tetsuro Imai ${ }^{2}$ \\ ${ }^{1}$ Faculty of Engineering, Niigata University, Niigata-shi, Niigata 950-2181, Japan \\ ${ }^{2}$ NTT DOCOMO, INC., 3-5 Hikari-no-oka, Yokosuka-shi, Kanagawa 239-8536, Japan \\ Correspondence should be addressed to Kentaro Nishimori, nishimori@ie.niigata-u.ac.jp
}

Received 1 June 2012; Revised 21 August 2012; Accepted 2 September 2012

Academic Editor: Ding-Bing Lin

Copyright (C) 2012 Kentaro Nishimori et al. This is an open access article distributed under the Creative Commons Attribution License, which permits unrestricted use, distribution, and reproduction in any medium, provided the original work is properly cited.

\begin{abstract}
This paper proposes interference-based decode and forward scheme that utilizes relay stations (RSs). In Long-Term-Evolution (LTE-) Advanced, heterogeneous networks in which femto- and picocells are overlaid onto macrocells are extensively discussed. However, interference between macro- and pico(femto)cells arises due to their different transmit power levels. Unlike conventional cooperative transmission schemes, the RS decodes interference in the first transmit timing period and forwards it to the user equipment (UE) in the second period. Moreover, cooperative transmission can be achieved without stopping the transmission from the base station (BS) to UE when forwarding the interference from the RS to the UE by utilizing the fact that signal-tonoise power ratio (SNR) between the RS and UE is much greater than that between the BS and UE. The basic performance of the proposed method is shown based on computer simulation. Moreover, the interference temperature and shadowing effect are measured when considering the coexistence between macro- and femtocells, and the performance of the proposed method is verified using measured shadowing effect.
\end{abstract}

\section{Introduction}

Due to the immense popularity of mobile phones and wireless LAN systems, increasing the data rate within a limited spectrum is one of the most important goals for wireless system design. Macrocells, the service areas which are one to several kilometers, were introduced in conventional cellular systems. On the other hand, femto- and picocells are currently the focus because small cells can enhance the frequency utilization and can be established using a low-powerconsuming base station (BS) that requires a small installation space. In Long-Term-Evolution (LTE-) Advanced, heterogeneous networks are extensively discussed in addition to traditional well-planned macrocell deployment to further improve the frequency utilization [1-3]. In heterogeneous network deployment, low power nodes such as femto-, pico, and relay nodes are placed throughout a macrocell layout, and they are placed generally in an unplanned manner. Hence, interference between macro- and pico(femto)cells occurs because the transmission power of pico(femto)cells is different from that of macrocell and the service coverage areas are different between these cells.

In this paper, we consider a macrocell system consisting of a BS and user terminals (UEs) and propose an interference-based decode and forward technique using a relay station (RS) to cancel the interference from pico- or femtocell. In recent years, cooperative transmission using RSs has been primarily used to improve the overall system capacity or to obtain a diversity gain $[4,5]$. On the other hand, RSs can also be utilized for interference cancellation $[6,7]$. The RS forwards information regarding the interference that is used by a receiver to decode the desired signal. Ideas on forwarding interference were considered in $[6,7]$ for the case when a destination node receives the desired signal and interference with small power, and instead of forwarding the desired signal, the RS forwards information regarding the interference. Moreover, interference cancellation techniques with the aid of the RS for cognitive radio are proposed $[8,9]$. However, the treatment in [6-9] is information-theoretic and did not explain the concrete procedure of how to use 
the RS in detail. In this work, we focus on a more practical setting with concrete system/modulation parameters, as well as a complete procedure for channel estimation, which is a prerequisite for actualizing distributed interference cancellation.

Interference-based decode and forward scheme using an RS for cognitive radio was proposed in [10]. In this method, the RS decodes and forwards the interference to a UE in a secondary system from a BS in the primary system using periodic training signals in the primary system. However, the time for interference transfer from the RS to UE is overhead in this method. We proposed a method that addresses this problem by utilizing the fact that the signal-to-noise ratio (SNR) between the RS and BS is greater than that between the BS and UE [11]. The optimal position of the relay stations by the proposed method is clarified based on computer simulation [11]. This paper brings significantly broadened treatment of the proposed method.

(i) The basic performance of the proposed method is evaluated in more detail. In particular, the proposed method is effective compared to the conventional adaptive array, regardless of the condition of SIR, SNRs of BS/RS and RS/UE.

(ii) Not only zero forcing (ZF) but also minimum mean square error (MMSE) algorithms with 2-element adaptive array at the UE are evaluated as the reference schemes, because MMSE algorithm is evaluated for the interference rejection between multicells for LTEAdvanced [12].

(iii) The performance of the proposed method is evaluated using measured data regarding a shadowing effect when considering coexistence between marco and femtocells.

When considering the RS as a virtual antenna which helps the UE from the interference, the configuration using the proposed method can be regarded as one of configurations regarding distributed antenna system.

The rest of the paper is organized as follows. Section 2 describes the target scenario and defines the problem to be addressed. Section 3 presents the proposed technique for interference cancellation using an RS. Section 4 provides quantitative performance analysis of the proposed scheme with theoretical propagation models. The affect on shadowing with the measured data is evaluated in Section 5. Section 6 concludes the paper.

\section{Target Scenario and Problem Definition}

Figure 1 shows a configuration for heterogeneous networks in LTE-Advanced [1]. In such heterogeneous networks, picocells, femtocells, and relays are deployed inside the service area of a macrocell in order to enhance the frequency utilization and guarantee the quality of service in high traffic areas. The picocell service area is several tens of meters. Femtocells have drawn much attention recently because their transmission power level is very low and thus they only require a low-power-consuming BS and small installation

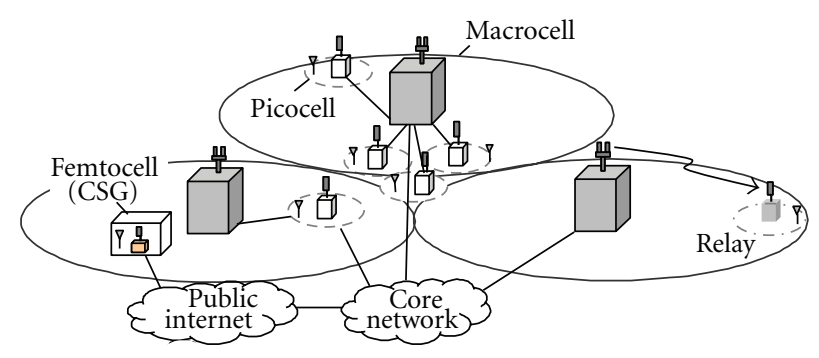

FIGURE 1: Configuration of heterogeneous network.

space. We focus on the coexistence between macro- and pico(femto)cells that use the same frequency band. However, the interference between macro- and pico(femto)cell occurs because the transmission power of pico(femto)cells is different from that of macrocells and the service coverage areas are different between macro- and pico(femto)cells. In this paper, we focus on the interference from a pico(femto) BS $(\mathrm{P}(\mathrm{F})-\mathrm{BS})$ to a set of macro-user equipment (M-UE), and we propose interference-based decode and forward scheme using RSs.

Figure 2 shows the signal format of the BS in macroand pico(femto)cells. Frequency division duplex (FDD) is generally used in macrocell BS (M-BS). On the other hand, pico- or femto-BS (P-BS or F-BS) employing time division duplex (TDD) is assumed in this paper. Both FDD and TDD are included as the standard of LTE-Advanced [13]. In such a scenario, P-BS transmits a signal using time slot 1 and receives a signal using time slot 2 as in Figure 2. In this paper, we utilize this feature in the proposed method.

\section{Proposed Method}

The proposed method cancels the interference at the M-UE from the P-BS by using the aid of as RS in the macrocell (MRS). Let us assume a downlink scenario. The RS with decode and forward generally receives the desired signal from the BS in time slot 1 and this signal is forwarded to the UE via the RS in the next time slot (time slot 2) $[4,5]$. Figure 3 shows the concept of the proposed method. Although three macrocells are considered as general system configuration in Figure 1, for the sake of ease, a macrocell is considered in Figure 3. In the proposed method, the M-RS decodes the interference from the P-BS (F-BS) at time slot 1 and the decoded interference is forwarded to the M-UE during time slot 2 when the interference from the P-BS (F-BS) arrives at the MUE. We assume that M-BS has multiple antennas but each RS has only one antenna in this paper. For simplification, $\mathrm{P}-\mathrm{BS}$ is treated as the interference hereafter but F-BS is also treated as interference in this paper. The transmit timing of the preamble signals for CSI estimation is shown in Figure 4. The proposed scheme can be explained in the following six steps.

(1) All CSI (M-BS/M-UE, M-BS/M-RE, M-RS/M-UE, P$\mathrm{BS} / \mathrm{M}-\mathrm{UE}$, and $\mathrm{P}-\mathrm{BS} / \mathrm{M}-\mathrm{RS}$ ) estimations are carried out using the preamble in Figure 4. 


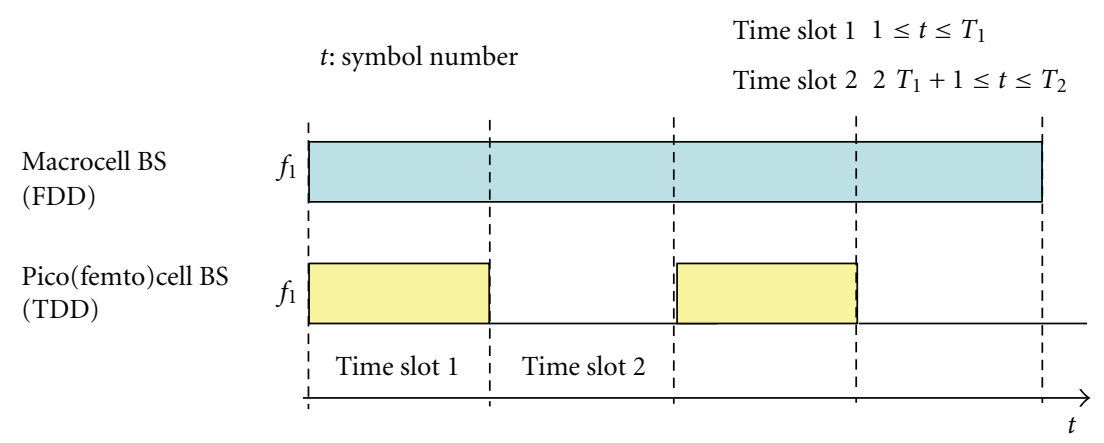

FIGURE 2: Signal format of base station in macro- and pico(femto)cells.

(2) The M-BS controls the transmit weight in order not to arrive the signal at the M-RS by using the CSI in Step 1 during the data period of time slot 1 .

(3) The interference incurred during the data period of time slot 1 is decoded using the received signal at the M-RS and the CSI from Step 1.

(4) The M-RS transmits the interference obtained in Step 3 to the M-UE during the next period of time slot 2 .

(5) The interference incurred during the data period for time slot 2 is estimated from the received signal at the $\mathrm{M}-\mathrm{UE}$ and the CSI from Step 1.

(6) The desired signals, $S_{1}(t)$ and $S_{2}(t)$, for the M-UE are estimated using the interference decoded in Step 5, the received signal in Steps 2 and 4, and the CSI from Step 1.

Steps 1 to 3 are employed during time slot 1 and Steps 4 to 6 are employed during time slot 2. In Step 1, we divide the period for the training sequence into three parts as shown in Figure 4 in order to estimate all the required CSIs. During Period 1, the M-BS and M-RS stop their transmissions to the M-UE such that only the CSI of the interference from the $\mathrm{P}-\mathrm{BS}$ to M-UE and M-RS is acquired. The received signals, $X_{P 1, U}(t)$ and $X_{P 1, R}(t)$, at the M-UE and M-RS during Period $1\left(1 \leq t \leq T_{p 1}, T_{p 1}=T_{p} / 3\right.$ in Figure 4$)$ are given below:

$$
\begin{gathered}
X_{P 1, U}(t)=h_{P U} I_{P 1}(t)+N_{U}(t), \\
X_{P 1, R}(t)=h_{P R} I_{P 1}(t)+N_{R}(t),
\end{gathered}
$$

where $h_{P U}$ and $h_{P R}$ denote the channel responses of the interfering signals for M-UE and M-RS, respectively. $T_{p}$ denotes the length of the preamble signal for P-BS. $I_{P 1}(t)$ represents the known preamble signals from the P-BS in Period 1. $N_{U}(t)$ and $N_{R}(t)$ represent the thermal noise for the M-UE and M-RS from the P-BS, respectively.

During Periods 2 and 3, signals are transmitted by the $\mathrm{M}-\mathrm{BS}$ and M-RS, respectively. The M-BS (M-RS) stops its transmission in Period 2 (3). The received signals, $X_{P 2, U}(t)$ and $X_{P 3, U}(t)$, at the M-UE for Period $2\left(T_{p 1}+1 \leq t \leq\right.$
$\left.T_{p 2}, T_{p 2}=2 T_{p 1}\right)$ and Period $3\left(T_{p 2}+1 \leq t \leq T_{p}\right)$ are given as

$$
\begin{aligned}
& X_{P 2, U}(t)=h_{B U} S_{P}(t)+h_{P U} I_{P 2}(t)+N_{U}(t), \\
& X_{P 3, U}(t)=h_{R U} S_{P}(t)+h_{P U} I_{P 3}(t)+N_{U}(t),
\end{aligned}
$$

where $h_{B U}$ and $h_{P U}$ denote the channel responses from the M-BS and M-RS to the M-UE, respectively. $I_{P 2}(t)$ and $I_{P 3}(t)$ represent the known preamble signals from P-BS in Periods 2 and 3, respectively. $S_{P}(t)$ denotes the known preamble signals from the M-BS and M-RS in Periods 2 and 3, respectively. During Period 2, M-RS receives the signal form M-BS and interference from P-BS. The received signals, $X_{P 2, R}(t)$, at the M-RS for Period 2 are given as

$$
X_{P 2, R}(t)=h_{B R} S_{P}(t)+h_{P R} I_{P 2}(t)+N_{R}(t),
$$

where $h_{B R}$ denotes the channel response from the M-BS to M-RS.

All CSIs are obtained using (1) to (5). The least square (LS) criteria is adopted for CSI estimation. The estimated CSIs of P-BS/M-UE and P-BS/M-RS are obtained using (1) and (2), respectively, as given below:

$$
\begin{aligned}
& \tilde{h}_{P U}=\frac{1}{T_{p 1}} \sum_{t=1}^{T p 1} \frac{X_{P 1, U}(t)}{I_{P 1}(t)}, \\
& \tilde{h}_{P R}=\frac{1}{T_{p 1}} \sum_{t=1}^{T p 1} \frac{X_{P 1, R}(t)}{I_{P 1}(t)} .
\end{aligned}
$$

The estimated CSIs of M-BS/M-UE, M-BS/M-RS and MRS/M-UE are obtained by using (3) and (7), respectively, as follows:

$$
\begin{aligned}
& \tilde{h}_{B U}=\frac{1}{T_{p 1}} \sum_{t=T p 1+1}^{T p 2} \frac{X_{P 2, U}(t)-\tilde{h}_{P U} I_{P 2}(t)}{S_{P}(t)}, \\
& \tilde{h}_{B R}=\frac{1}{T_{p 1}} \sum_{t=T p 1+1}^{T p 2} \frac{X_{P 2, R}(t)-\tilde{h}_{P R} I_{P 2}(t)}{S_{P}(t)}, \\
& \tilde{h}_{R U}=\frac{1}{T_{p 1}} \sum_{t=T p 2+1}^{T p 3} \frac{X_{P 3, U}(t)-\tilde{h}_{P U} I_{P 3}(t)}{S_{P}(t)} .
\end{aligned}
$$




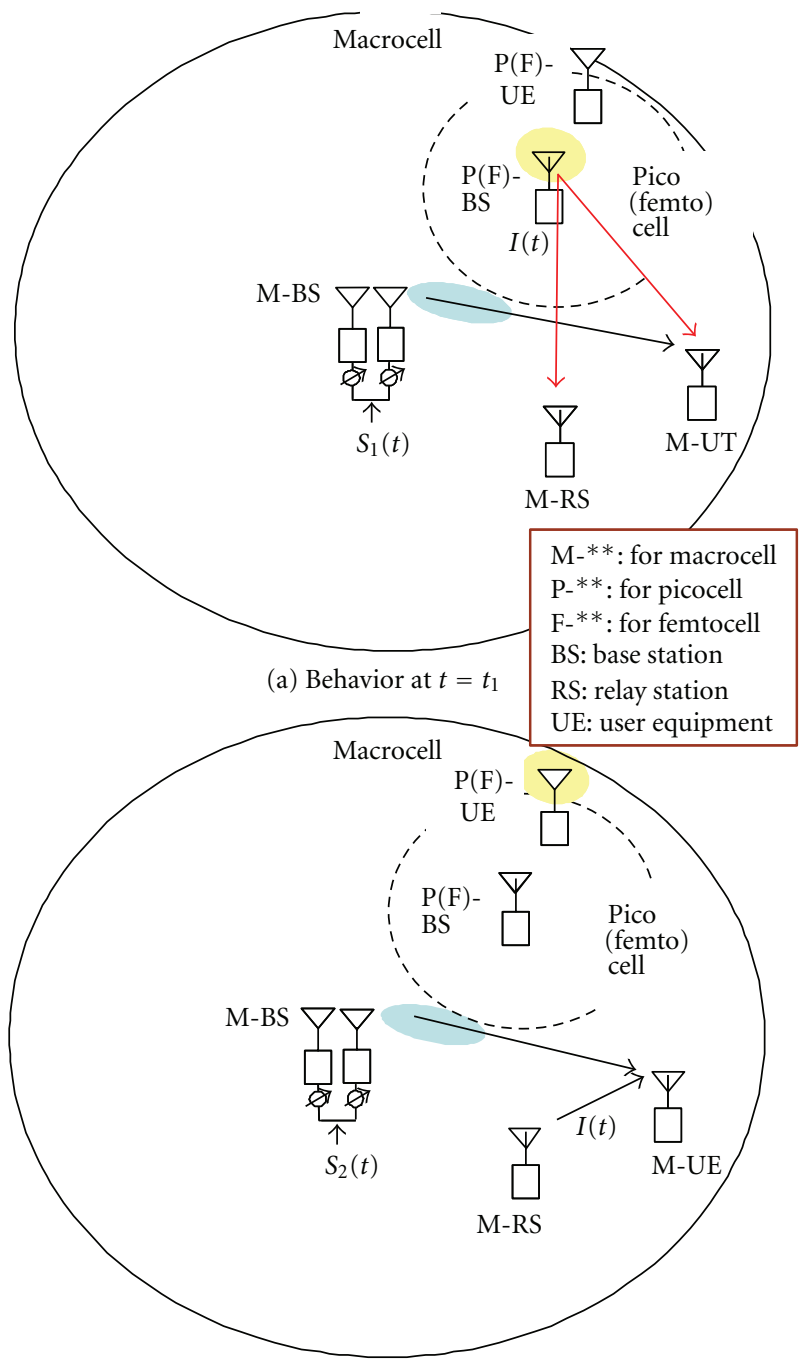

(b) Behavior at $t=t_{2}$

FIGURE 3: Concept of proposed method.

As shown in (6) to (10), $T_{p 1}$ is required as the number of symbols for the channel estimation. Throughout the evaluation, $T_{p 1}$ is set to be five.

The propagation channel between M-BS and M-RS is not basically changed because these stations do no move, unlike M-UE. Hence, we can consider that the propagation channel is not changed between Steps 1 and 2. When considering the channel between M-BS and MUE, the adaptive weight may be changed due to the Doppler shift. On the other hand, transmission speed for one time slot can be assumed to be much shorter than the Doppler frequency when considering recent broadband wireless communication. However, when the Doppler frequency is very high, the adaptive weight might be changed. We must consider this issue as one of future works.

In the second step (Step 2), the M-BS transmits a signal to the M-UE with an antenna pattern that creates a null toward the M-RS during the data period for time slot 1 in Figure 3. The received signals at the M-UE and M-RS are expressed as

$$
\begin{gathered}
X_{t_{1}, U}(t)=h_{B U} S_{1}(t)+h_{P U} I(t)+N_{U}(t), \\
X_{t_{1}, R}(t)=h_{P R} I(t)+N_{R}(t),
\end{gathered}
$$

where $S_{1}(t)$ and $I(t)$ represent unknown data signals for the $\mathrm{M}-\mathrm{UE}$ from the M-BS and P-BS, respectively. Hence, the MUE must decode and cancel interference $I(t)$ without any information pertaining to the interference.

$S_{1}(t)$ cannot be decoded using the procedure in Steps 1 and 2. On the other hand, interference $I(t)$ can be estimated at the M-RS using (12) and $\tilde{h}_{P R}$ obtained in Step 1. In Step 3, the M-RS decodes the interference that occurs during the data period of time slot 1 . The decoded interference, $\tilde{I}(t)$, at the M-RS is obtained using (7) and (12) as

$$
\begin{aligned}
\widetilde{I}(t) & =\frac{X_{t_{1}, R}(t)}{\widetilde{h}_{P R}} \\
& =\frac{h_{P R}}{\widetilde{h}_{P R}} I(t)+\frac{N_{R}(t)}{\tilde{h}_{P R}} \\
& \approx I(t)+\frac{N_{R}(t)}{\tilde{h}_{P R}} .
\end{aligned}
$$

Here, interference-to-noise power ratio (INR) at the M-RS is $E\left[\left|h_{P R}\right|^{2} /\left|N_{R}(t)\right|^{2}\right]$. When the INR is large, the second term on the right side of (13) is negligible. In Step 4, the M-RS transmits interference $\widetilde{I}(t)$ estimated in Step 3 to the M-UE using the next data period for time slot 2 in Figure 3. The M-BS transmits a signal to the M-UE using this period. The received signal at the $\mathrm{M}-\mathrm{UE}$ is denoted by

$$
X_{t_{2}, U}(t)=h_{B U} S_{2}(t)+h_{R U} \tilde{I}(t)+N_{U}(t),
$$

where $S_{2}(t)$ represents unknown data signals for the M-UE from the M-BS at time slot 2.

In Step 5, after the transmission of the interference, the interference from the P-BS during the data period for time slot $1, \tilde{I}(t)$, is estimated by using the received signal in (14). First, (14) is changed using (10) as:

$$
\frac{X_{t_{2}, U}(t)}{\widetilde{h}_{R U}}=\frac{h_{B U}}{\tilde{h}_{R U}} S_{2}(t)+\frac{h_{R U}}{\tilde{h}_{R U}} \tilde{I}(t)+\frac{N_{U}(t)}{\tilde{h}_{R U}} .
$$

When $h_{R U} \approx \tilde{h}_{R U}$, signal-to-interference power ratio (SIR) and INR at M-UE are denoted as $E\left[\left|h_{B U}\right|^{2} /\left|h_{R U}\right|^{2}\right]$ and $E\left[\left|h_{R U}\right|^{2} /\left|N_{U}(t)\right|^{2}\right]$, respectively. It is reasonable to assume that $E\left[\left|h_{R U}\right|^{2}\right]$ is much larger than $E\left[\left|h_{B U}\right|^{2}\right]$ and $E\left[\left|N_{U}(t)\right|^{2}\right]$, because the M-RS should be located near the M-UE. In other word, the SIR is very small and the INR is very large. Hence, the first and third terms on the right side of (15) can be approximately assumed to be much smaller than the second term on the right side of (15). Note that SNR, $E\left[\left|h_{B U}\right|^{2} /\left|N_{U}(t)\right|^{2}\right]$ is not small and the first term on the right side of (15) is not zero. As a result, when we ignore the first and third terms on the right side of (15), (15) can be approximated as

$$
\frac{X_{t_{2}, U}(t)}{\widetilde{h}_{R U}} \approx \widetilde{I}(t) \equiv \hat{I}(t) .
$$




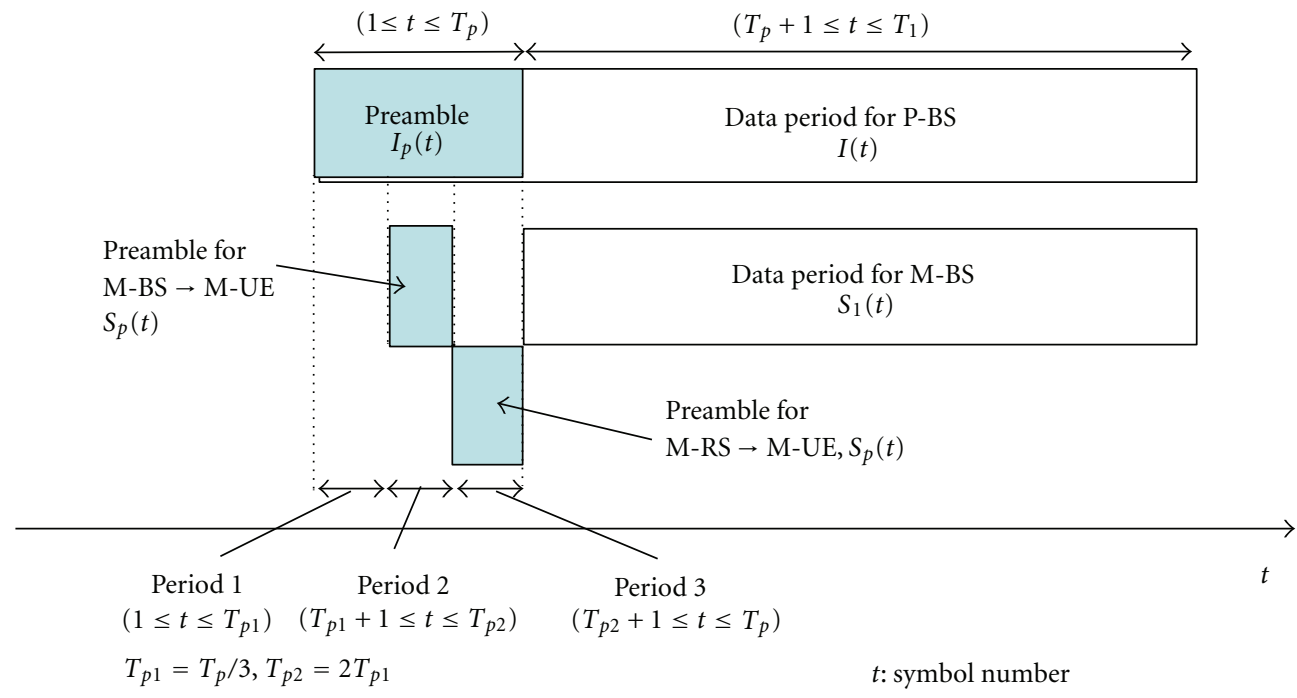

FIGURE 4: Transmit timing of preamble signals for CSI estimation.

In Step 6, the desired signals, $S_{1}(t)$ and $S_{2}(t)$, can be estimated using interference $\widehat{I}(t)$ estimated in Step 5 , received signals $X_{t_{1}, U}(t)$ and $X_{t_{2}, U}(t)$ in Steps 2 and 4 , and CSIs $\tilde{h}_{B U}$, $\tilde{h}_{P U}$, and $\widetilde{h}_{R U}$ obtained in Step 1 . The estimated desired signals, $\widetilde{S}_{1}(t)$ and $\widetilde{S}_{2}(t)$, can be denoted as.

$$
\begin{aligned}
& \tilde{S}_{1}(t)=\frac{X_{t_{1}, U}(t)-\tilde{h}_{P U} \widehat{I}(t)}{\widetilde{h}_{B U}}, \\
& \tilde{S}_{2}(t)=\frac{X_{t_{2}, U}(t)-\widetilde{h}_{R U} \widehat{I}(t)}{\widetilde{h}_{B U}} .
\end{aligned}
$$

\section{Basic Characteristics of the Proposed Method}

In this section, we evaluate the performance of the proposed method by computer simulation. Figure 5 represents the configurations of the proposed and reference scheme. We compare the proposed interference cancellation method (Method (A)) to a reference scheme (Method (B)), an adaptive array using ZF/MMSE. For a fair comparison, the total number of antennas at the transmitter and receiver sites are identical for Methods (A) and (B). The numbers of antennas at the M-BS and the M-UE are two, respectively, in Method (B). The number of antennas at the M-BS is two and that at the M-RS plus M-UE is also two in Method (A). In Method (B), the M-UE uses an adaptive array with ZF/MMSE [12]. When using the ZF algorithm, the decoded signal is denoted as

$$
\begin{aligned}
\left(\begin{array}{c}
\tilde{S}(t) \\
\widetilde{I}(t)
\end{array}\right) & =\left(\begin{array}{ll}
h_{S_{1}} & h_{I_{1}} \\
h_{S_{2}} & h_{I_{2}}
\end{array}\right)^{-1}\left(\begin{array}{l}
X_{U_{1}} \\
X_{U_{2}}
\end{array}\right) \\
& =\left(\begin{array}{ll}
\mathbf{h}_{S} & \mathbf{h}_{I}
\end{array}\right)^{-1} \mathbf{X}_{U} \\
& =\mathbf{H}^{-1} \mathbf{X}_{U},
\end{aligned}
$$

where $\mathbf{h}_{S}=\left[h_{S_{1}}, h_{S_{2}}\right]^{T}$ and $\mathbf{h}_{I}=\left[h_{I_{1}}, h_{I_{2}}\right]^{T}$ denote the channel responses for the M-UE from the M-BS and P-BS, respectively. $\mathbf{X}_{U}=\left[X_{U_{1}}, X_{U_{2}}\right]^{T}$ is the received signal vector at the M-UE. When using the MMSE algorithm, the decoded signal is denoted as

$$
\left(\begin{array}{c}
\widetilde{S}(t) \\
\widetilde{I}(t)
\end{array}\right)=\left(\mathbf{H}^{H} \mathbf{H}+\sigma^{2} \mathbf{U}\right)^{-1} \mathbf{H}^{H} \mathbf{X}_{U}
$$

where $\mathbf{U}$ is $2 \times 2$ identity matrix and $\sigma^{2}$ is the noise power. A total transmit power by M-BS and M-RS in the proposed method is the same with that by M-BS in the conventional method.

Transmissions from the M-BS to M-UE in the macrocell use adaptive modulation. Table 1 gives the required SNRs for different modulation levels. The SNR for each modulation scheme is given in Table 1 for the bit error rate (BER) of $5 \times 10^{-3}$ [14]. For all the methods, the modulation level is decided based on the instantaneous SNR. The modulation scheme for the interference is QPSK.

The total transmission rate, $R$, is defined as

$$
\begin{gathered}
R=\frac{\sum_{k=1}^{N_{F}} R(k)}{N_{F}}, \\
R(k)=M_{a}[1-\operatorname{BER}(k)],
\end{gathered}
$$

where $N_{F}$ represents the number of frames. $M_{a}$ denotes the number of bits per symbol when applying the adaptive modulation. $R(k)$ and $\operatorname{BER}(k)$ are the transmission rate and BER for the $k$ th frame, respectively. In the simulation, we set the length per frame to 600 and $N_{F}=20000$. A Rayleigh fading environment is assumed among all nodes. In the evaluation, we did not consider channel codes in order to evaluate the basic ability of the proposed method.

First, the transmission rate is evaluated versus the SIR in Figure 6, in order to confirm the effectiveness of the approximation in (16). Here, we assume that the desired 

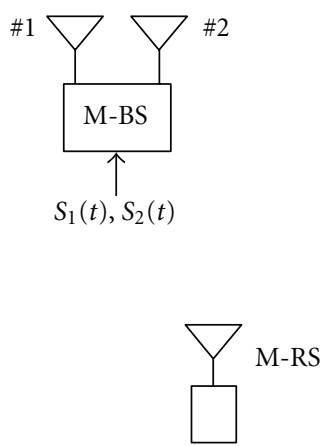

P-BS

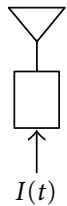

(a) Configuration for Method (A) Method (A): Proposed method
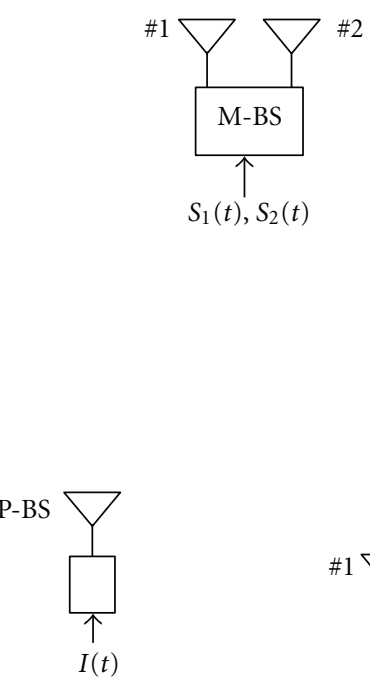

(b) Configuration for Method (B) Method (B): Adaptive array using ZF/MMSE algorithms

FIgure 5: Proposed and reference schemes.

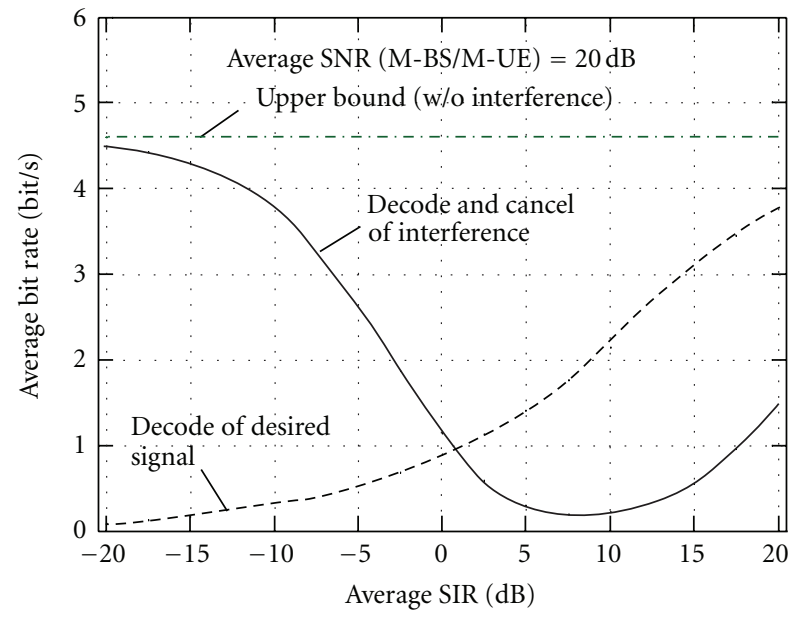

FIgURe 6: Transmission rate versus SIR.

signal $(S)$ and the interference $(I)$ are the signals from the $\mathrm{M}-\mathrm{BS}$ and M-RS to the M-UE at time slot 2 in Figure 3, respectively. For comparison, the results when only the desired signal is decoded are shown. Figure 6 shows that the transmission rate is improved when the SIR is decreased. When the SIR is less than $-10 \mathrm{~dB}$, the degradation compared to the transmission rate with the upper bound is less than $1 \mathrm{bit} / \mathrm{s}$. Hence, the approximation in (16) is effective when considering the SNR between the M-RS and M-UE to be greater than that between the M-BS and M-UE.

The SNR between the M-RS and M-UE is a very important parameter that determines the ability for decoding the interference. The transmission rates of the proposed method versus the SNR between the M-RS and M-UE are plotted in Figure 7. The average SNR between the M-BS and M-UE and the SIR between the M-BS and P-BS for

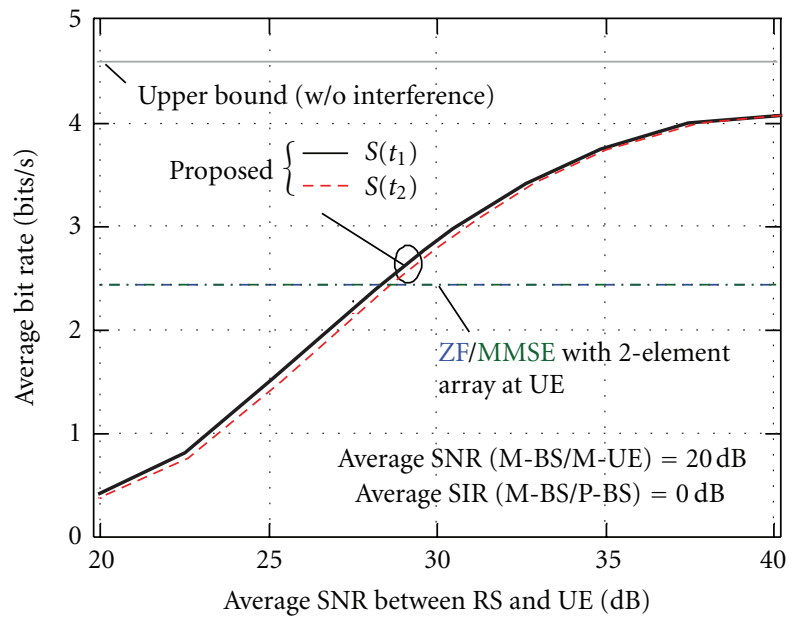

Figure 7: Transmission rate versus SNR between M-RS and M-UE.

the M-UE are set to 20 and $0 \mathrm{~dB}$, respectively. The average transmission rate for time slots 1 and 2 is shown in Figure 7. As shown in Figure 7, the transmission rates for time slots 1 and 2 are improved according to the SNR between the $\mathrm{M}-\mathrm{RS}$ and M-UE and both transmission rates are almost the same. Moreover, the proposed method obtains a higher transmission rate than that for 2-element adaptive array using ZF/MMSE algorithm when the SNR between the MRS and M-UE is greater than $28 \mathrm{~dB}$. Note that the average bit rate by the MMSE algorithm is almost same with that by the $\mathrm{ZF}$ algorithm. The proposed method is effective compared to the adaptive array with ZF/MMSE when the SNR between the M-RS and M-UE is $8 \mathrm{~dB}$ higher than that between the $\mathrm{M}-\mathrm{BS}$ and M-UE.

Figure 8 plots the transmission rates of the proposed method and ZF/MMSE adaptive array (Method (B)) versus 


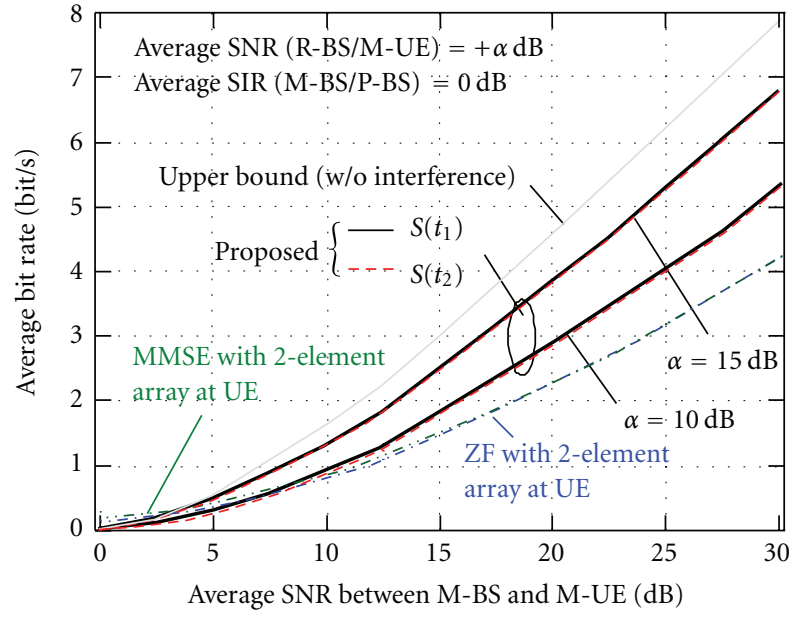

FIGURE 8: Transmission rate versus SNR between M-RS and M-UE.

the SNR between the M-BS and M-UE. Note that the average bit rate by the MMSE algorithm is almost the same with that by the ZF algorithm except very small SNR. The results are plotted when the SNR between the M-RS and M-UE is $10 / 15 \mathrm{~dB}$ ( $\alpha$ in Figure 8), greater than that between the M-BS and M-UE. As shown in the figure, by applying the proposed method, the transmission rate is improved compared to that for Method (B), when the SNR between the B-RS and M-UE is greater than 10 and $7 \mathrm{~dB}$, respectively, and when $\alpha$ is 10 and $15 \mathrm{~dB}$.

\section{Performance Evaluation Considering Coexistence between Macro- and Femtocells Using Measured Shadowing Effect}

In this section, the performance of the proposed method is evaluated when considering the coexistence between macro and femtocells using measured shadowing effect. Since there are UEs that do not belong to a closed subscriber group (CSG) BS in a femtocell, such UEs might give/receive interference to/from the femtocell [1]. Moreover, the femtocell is generally used in indoor scenarios, and the propagation characteristics between the femto- and macrocells are different from those inside a macrocell. We performed measurements to obtain the shadowing characteristics between femto- and macrocells and inside a macrocell and reflected the results in a computer simulation.

Figure 9 shows the considered measurement environment. The measurements are taken in the Faculty of Engineering building of Niigata University in Japan. The MUE is located in a parking area and moved around Courses 1 to 10 in Figure 9. The M-BS and F-BS are located at the top of the building and the 4 th floor in Figure 9, respectively. The antenna heights of the M-BS and F-BS are 22.5 and $16.5 \mathrm{~m}$, respectively. The height of the M-UE is $1 \mathrm{~m}$. The F-BS is located in the place in Figure 10, because the influence of the shadowing might be changed by the difference of the place in Figure 10 . The radio frequency is $2.2 \mathrm{GHz}$ and a continuous wave $(10 \mathrm{~W})$ is transmitted from the M-BS or F-BS to
TABLE 1: Required SNR for different modulation levels.

\begin{tabular}{lc}
\hline SNR & Modulation \\
\hline $3.63 \mathrm{~dB}$ & BPSK \\
$8.40 \mathrm{~dB}$ & QPSK \\
$12.08 \mathrm{~dB}$ & 8QAM \\
$15.39 \mathrm{~dB}$ & $16 \mathrm{QAM}$ \\
$18.55 \mathrm{~dB}$ & $32 \mathrm{QAM}$ \\
$21.63 \mathrm{~dB}$ & 64QAM \\
$24.67 \mathrm{~dB}$ & 128QAM \\
$27.70 \mathrm{~dB}$ & 256QAM \\
$30.72 \mathrm{~dB}$ & 512QAM \\
$33.73 \mathrm{~dB}$ & 1024QAM \\
\hline
\end{tabular}

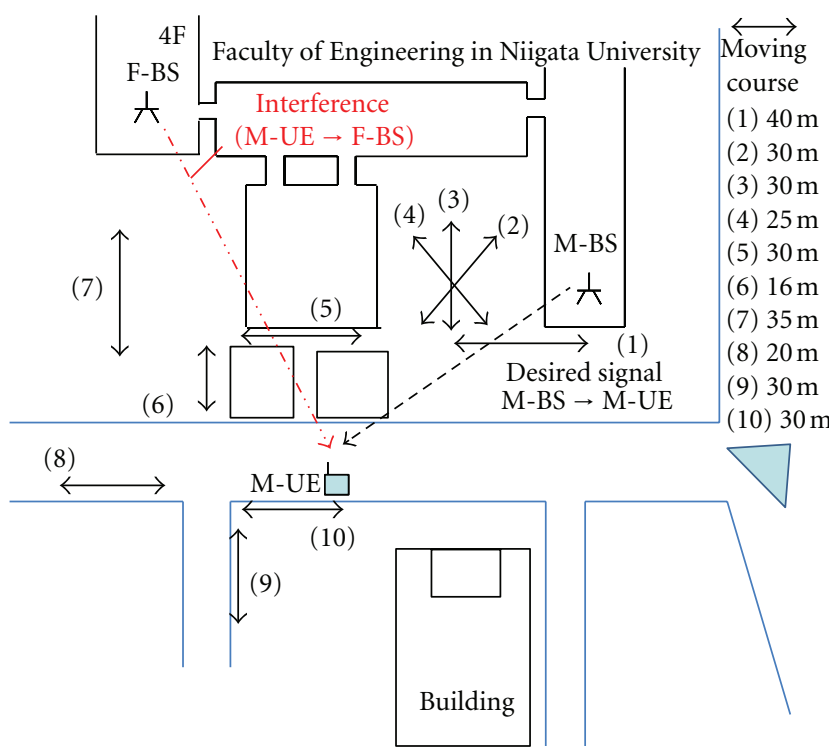

Figure 9: Measurement environment.

the M-UE. The received power and shadowing effect are measured. The shadowing effect is obtained by calculating the standard deviation of median values in the received power. The median values are obtained with the distance of $1 \mathrm{~m}$. We assume the scenario where the interference from the femtocell arrives at the M-UE when the M-BS communicates with the M-UE.

Figure 11 denotes the received power for the desired signal (M-BS/M-UE, outdoor) and interference (F-BS/MUE, Cases 1, 2, and 3) when considering Courses 1 to 10. Table 2 show the standard deviation of the shadowing effect for the M-BS/M-UE and F-BS/M-UE, respectively. As shown in Figure 11, the power of desired signal is $3 \mathrm{~dB}$ higher than that of interference (Case 1) when considering CDF = $50 \%$. Due to the influence of penetration loss in indoor, the received powers by Case 2 and 3 are lower than that by Case 1. Table 2 shows that the standard deviation of the shadowing is for the desired signal 5.1 and from 4.8 to 6.6 for the interference, respectively. Since outdoor-to-indoor propagation is considered when considering the interference from/to the femtocell to/from the M-UE, the range of the 


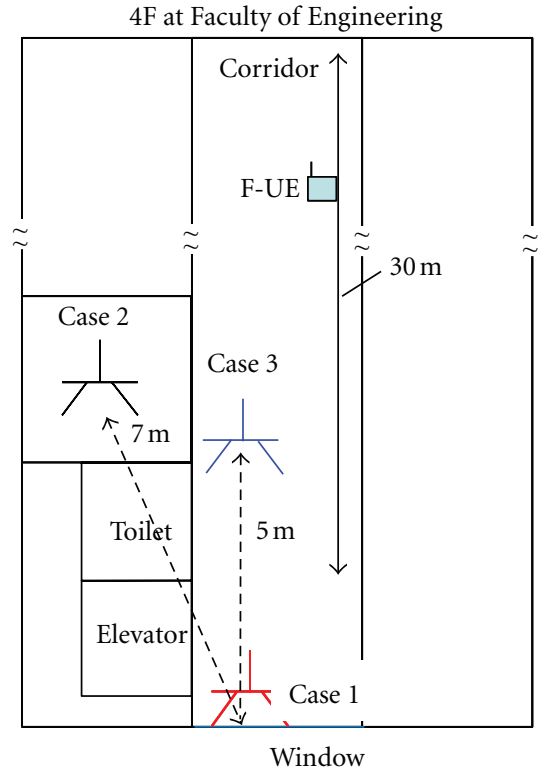

FIGURE 10: Location of femto-BS.

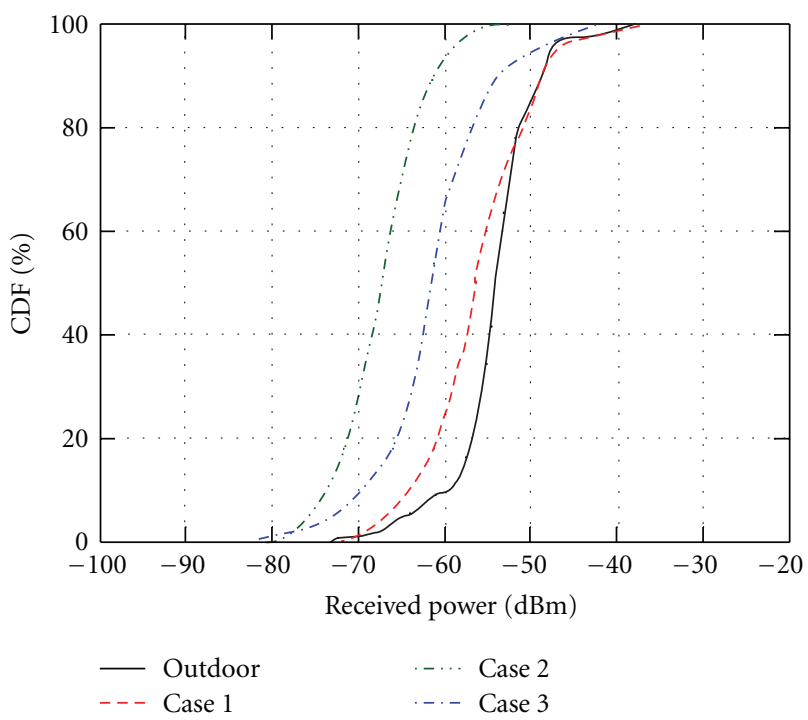

FIGURE 11: Received powers for desired signal and interference. Outdoor: desired signal, interference: Cases 1 to 3.

TABLE 2: Standard deviation of shadowing.

\begin{tabular}{lc}
\hline Type & Standard deviation $[\mathrm{dB}]$ \\
\hline Outdoor (M-BS/M-UE) & 5.1 \\
Case 1 (F-BS/M-UE) & 6.1 \\
Case 2 (F-BS/M-UE) & 4.8 \\
Case 3 (F-BS/M-UE) & 6.6 \\
\hline
\end{tabular}

standard deviation for the F-BS/M-UE is larger than that for the M-BS/M-UE.

The transmission bit rate is evaluated using the measured standard deviation of the shadowing. Table 3 gives the simulation parameters. Considering the value on the standard
TABLE 3: Simulation parameters.

\begin{tabular}{lccc}
\hline Parameter & Macro-BS & Pico-BS & Relay \\
\hline Cell radius & $2312 \mathrm{~m}$ & $40 \mathrm{~m}$ & - \\
BS Tx power & $46 \mathrm{dBm}$ & $30 \mathrm{dBm}$ & $30 \mathrm{dBm}$ \\
UE Tx power & $27 \mathrm{dBm}$ & $27 \mathrm{dBm}$ & - \\
BS ant. gain & $14 \mathrm{dBi}$ & $5 \mathrm{dBi}$ & $5 \mathrm{dBi}$ \\
UE ant. gain & $0 \mathrm{dBi}$ & $0 \mathrm{dBi}$ & - \\
Noise power & $-174 \mathrm{dBm} / \mathrm{Hz}$ & - & $-174 \mathrm{dBm} / \mathrm{Hz}$ \\
Antenna height & $22.5 \mathrm{~m}$ & $16.5 \mathrm{~m}$ & $15 \mathrm{~m}$ \\
Path loss $(\rightarrow$ UE) & C2 $($ NLOS $)$ & B1 $(\mathrm{LOS})$ & B1 $(\mathrm{LOS})$ \\
\hline
\end{tabular}

deviation of shadowing, the results of outdoor and Case 2 in Table 2 are used for M-BS/M-UE and F-BS/M-UE transmissions, respectively. Figure 12 denotes the locations of M-BS, M-UE, F-BS, and M-RS. Three femtocells are assumed to be located inside a macrocell. F-BSs are randomly located inside the service area in M-BS. M-UE is also located inside the service area in M-BS. We employed the simulation where the location on F-BSs and M-UE is changed. The trial number is 10000 . Regarding the basic parameter in Table 3, we refer to the parameters which are used in [2]. Regarding the cell radius of M-BS, when the cell radius is $289 \mathrm{~m}$ [2], we assume the cell size of 8 times, because the effectiveness of the relay station should be evaluated.

Since the evaluation using heterogeneous path loss conditions is essential when considering heterogeneous networks $[15,16]$, the propagation pathloss model proposed in [17] is used in this paper. Model C2, which is used for typical macrocell non-line-of-sight (NLOS) scenarios, is adopted for the path loss between the M-BS and M-UE. Since we consider the interference from the F-BS to M-UE, Model B1, which is used for microcell line-of-sight (LOS) scenarios, is adopted for the path loss between the P-BS and M-UE. Model B1 is also used for the pathloss between the M-RS and M-UE. The path loss between the M-BS and MRS is Model B5f (NLOS), which is used for relay transmission between the M-BS and M-RS. The relay stations are located at the position of $d / 2$ in Figure 12. Four relay stations are assumed in Figure 12. When considering the propagation of $\mathrm{M}-\mathrm{BS} / \mathrm{M}-\mathrm{RS}, \mathrm{M}-\mathrm{RS} / \mathrm{M}-\mathrm{UE}$, and F-BS/M-RS, the values on the standard deviation of shadowing are used in those in [17]. The number of relays, $N_{R}$, is changed from 4 to 16 in a circular arrangement. The other simulation conditions are the same as those described in Section 4. We assume that the M-UE and M-RS can exchange the information with a certain interval, and that the M-UE can ideally select M-RS from which SNR to M-UE is the highest. We evaluate average transmission rate which means average cell throughput of MBS. In the evaluation, we did not consider channel codes in order to evaluate the basic ability of the proposed method.

In the simulation, we assume that the M-RS obtains the information regarding the adaptive modulation scheme by using the control channel of F-BS, because the M-RS can receive only interference by the F-BS. Moreover, we assume that the M-RS correctly informs the information regarding adaptive modulation scheme by the F-BS to the M-UE. 


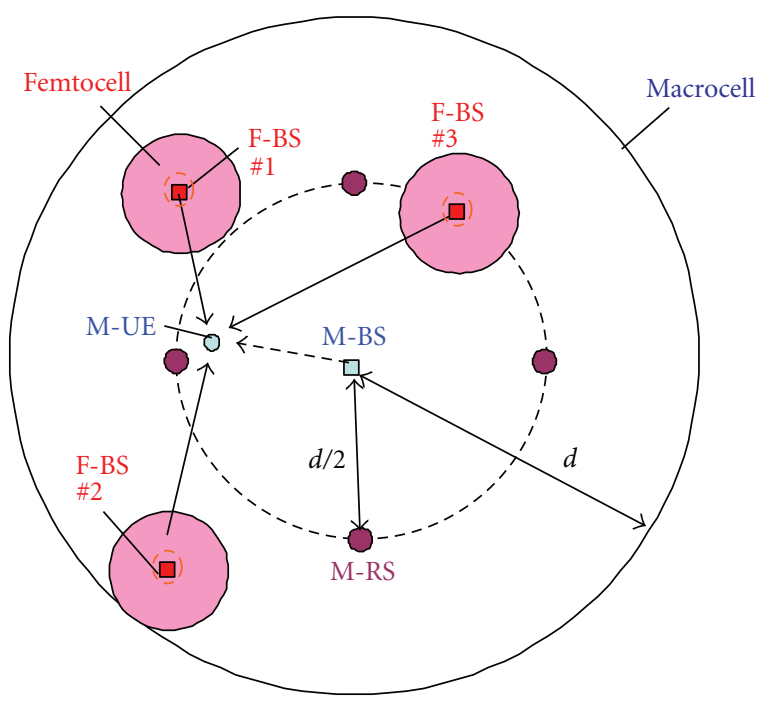

Figure 12: Location of M-BS, M-UE, F-BS, and M-RS.

Figure 13 shows the relationship between the transmission rate versus the number of relays, $N_{R}$. Not only QPSK modulation but also adaptive modulation for F-BS is assumed in Figure 13. The figure shows that the transmission rate is improved when $N_{R}$ is increased, but the performance becomes gradually saturated when $N_{R} \geq 6$ when considering only QPSK modulation for F-BS. Although the transmission rate is degraded compared to the case with only QPSK modulation for F-BS when assuming adaptive modulation for F-BS, we confirm that the transmission rate is gradually increased by the aid of increasing number of relays. The reason why the average bit rate (of $\mathrm{M}$-UE) decreases when F-BS is allowed to use adaptive modulation is that the performance by the proposed method depends on the interference decoding in (13) at the M-RS. When the higher modulation scheme in F-BS is employed, the higher interference-tonoise power Ratio (INR) is required at the M-RS for the interference decoding. Moreover, it is shown that the proposed method outperforms the ZF/MMSE schemes even when $N_{R}=4$. The average bit rate by the MMSE algorithm is almost the same with that by the ZF algorithm.

\section{Conclusion}

This paper proposed interference-based decode and forward method using the RSs in heterogeneous networks. The RS in a macrocell system decodes and forwards the interference from the pico(femto)cell to the UE, and the UE can decode the signal without stopping the signal reception from the BS by utilizing the higher SNR between the RS and UE compared to that between BS and UE in the proposed method. We clarified the effectiveness of the proposed method compared to using the conventional adaptive array with ZF and MMSE algorithms when the SNR between the RS and UE is $8 \mathrm{~dB}$ greater than that between the BS and UE. Moreover, we confirmed the effectiveness of the proposed method based on evaluation using the measured data for

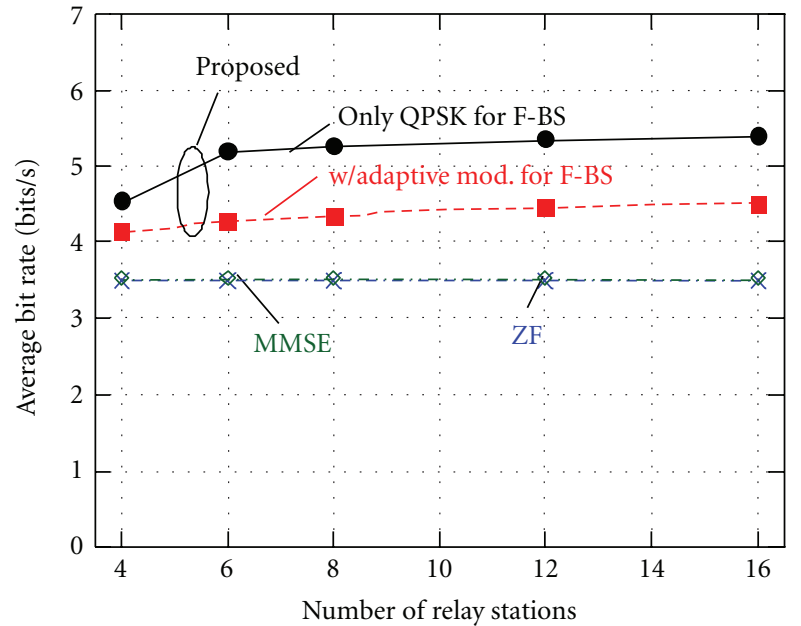

FIGURE 13: Transmission rate versus transmit power of F-BS when considering shadowing effect.

shadowing effects when considering the coexistence between macro- and femtocells.

As for the future work, we evaluate the influence when changing the service area of macrocell/picocell, because the interference power is largely affected by the service area of macrocell/picocell. In this paper, our aim was to propose the basic technique when the relay nodes can be applied and we focused on the basic ability of the interference cancellation by using the proposed method. On the other hand, hybrid ARQ is adopted as one of the important techniques in LTEAdvanced and the evaluation by using the proposed method including the channel codes is essential for the future work.

\section{References}

[1] 3GPP, TR 36.814 (V9.0.0), "Further advancements for EUTRA physical layer aspects," 2010.

[2] M. Tanno, A. Morimoto, T. Abe, Y. Kishiyama, and T. Nakamura, "Heterogeneous Network in LTE-Advanced," IEICE Technical Report RCS2009-317, 2010.

[3] A. Khandekar, N. Bhushan, J. Tingfang, and V. Vanghi, "LTEadvanced: heterogeneous networks," in European Wireless Conference, pp. 978-982, April 2010.

[4] R. U. Nabar, H. Bölcskei, and F. W. Kneubühler, "Fading relay channels: performance limits and space-time signal design," IEEE Journal on Selected Areas in Communications, vol. 22, no. 6, pp. 1099-1109, 2004.

[5] K. Yamamoto, H. Maruyama, T. Shimizu, H. Murata, and S. Yoshida, "Spectral efficiency of fundamental cooperative relaying in interference-limited environments," IEICE Transactions on Communications, vol. 91, no. 8, pp. 2674-2682, 2008.

[6] R. Dabora, I. Maric, and A. Goldsmith, "Interference forwarding in multiuser networks," in Proceedings of the IEEE Global Telecommunications Conference, December 2008.

[7] I. Marié, R. Dabora, and A. Goldsmith, "On the capacity of the interference channel with a relay," in Proceedings of the IEEE International Symposium on Information Theory (ISIT '08), July 2008. 
[8] A. Jovičić and P. Viswanath, "Cognitive radio: an informationtheoretic perspective," IEEE Transactions on Information Theory, vol. 55, no. 9, pp. 3945-3958, 2009.

[9] P.-H. Lin, S.-C. Lin, C.-P. Lee, and H.-J. Su, "Cognitive radio with partial channel state information at the transmitter," IEEE Transactions on Wireless Communications, vol. 9, no. 11, pp. 3402-3413, 2010.

[10] K. Nishimori, H. Yomo, P. Popovski, Y. Takatori, R. Prasad, and S. Kubota, "Distributed interference cancellation for dynamic spectrum sharing," in Proceedings of the IEEE International Conference on Communications (ICC '08), pp. 30443049, May 2008.

[11] K. Nishimori, K. Kitao, and T. Imai, "Interference cancellation using relay station in heterogeneous networksin," in Proceedings of the IEEE Vehicular Technology Conference (VTC Fall '11), September 2011.

[12] Y. Ohwatari, N. Miki, T. Asai, T. Abe, and H. Taoka, "Performance of advanced receiver employing interference rejection combining to suppress inter-cell interference in LTE-advanced downlink," in Proceedings of the IEEE Vehicular Technology Conference (VTC Fall '11), September 2011.

[13] 3GPP, TS 36.201 (V10.0.0), Evolved universal terrestrial radio access (E-UTRA), LTE physical layer, General description (Release 10), 2010.

[14] S. T. Chung and A. J. Goldsmith, "Degrees of freedom in adaptive modulation: a unified view," IEEE Transactions on Communications, vol. 49, no. 9, pp. 1561-1571, 2001.

[15] K. Nishimori, R. D. Taranto, H. Yomo et al., "Spatial opportunity for cognitive radio systems with heterogeneous path loss conditions," in Proceedings of the IEEE 65th Vehicular Technology Conference (VTC '07), Dublin, Ireland, April 2007.

[16] K. Nishimori, R. Di Taranto, H. Yomo, and P. Popovski, “Cognitive radio operation under directional primary interference and practical path loss models," IEICE Transactions on Communications, vol. 94, no. 5, pp. 1243-1253, 2011.

[17] WINNER II channel models, http://www.ist-winner.org/deliverables.html. 

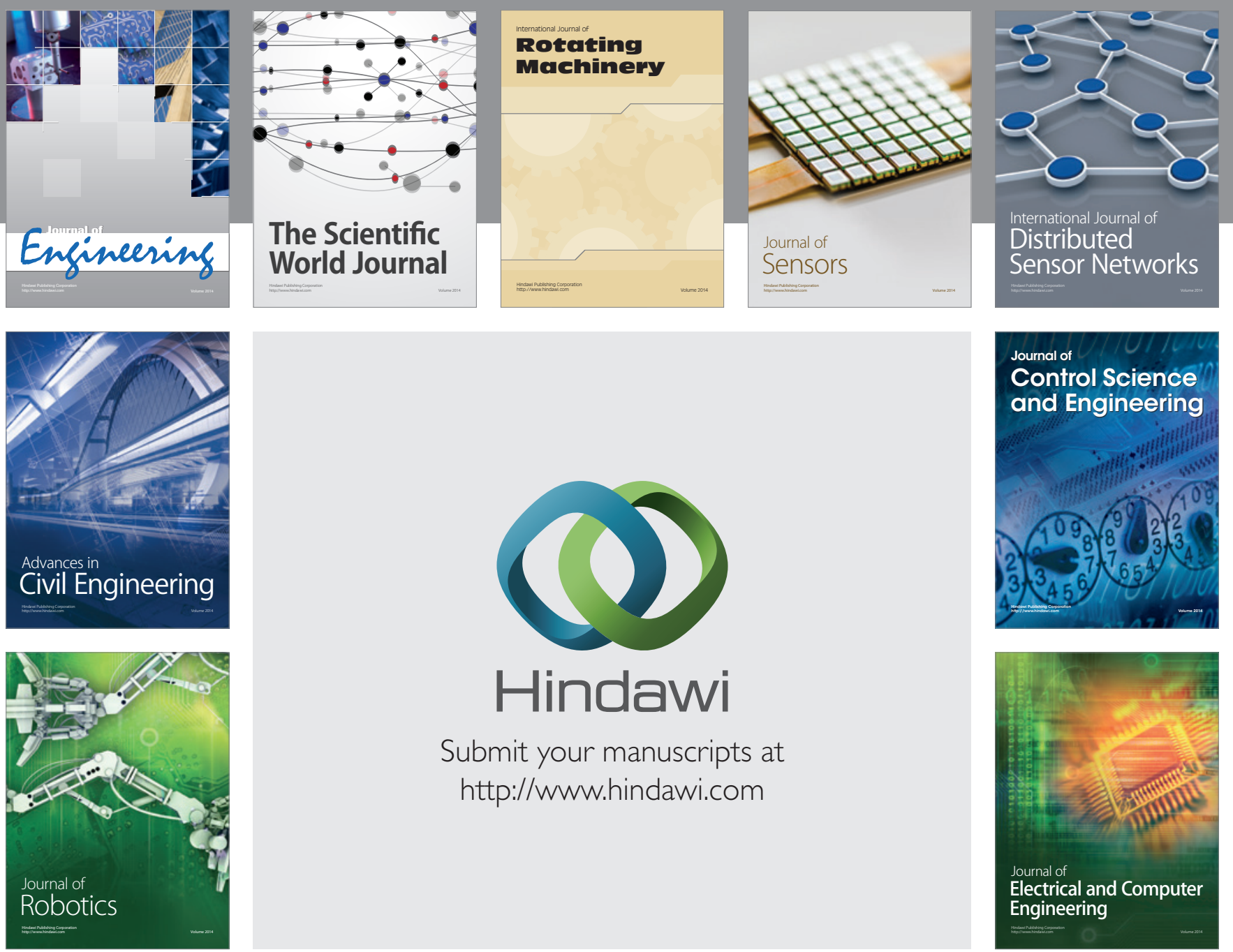

Submit your manuscripts at

http://www.hindawi.com
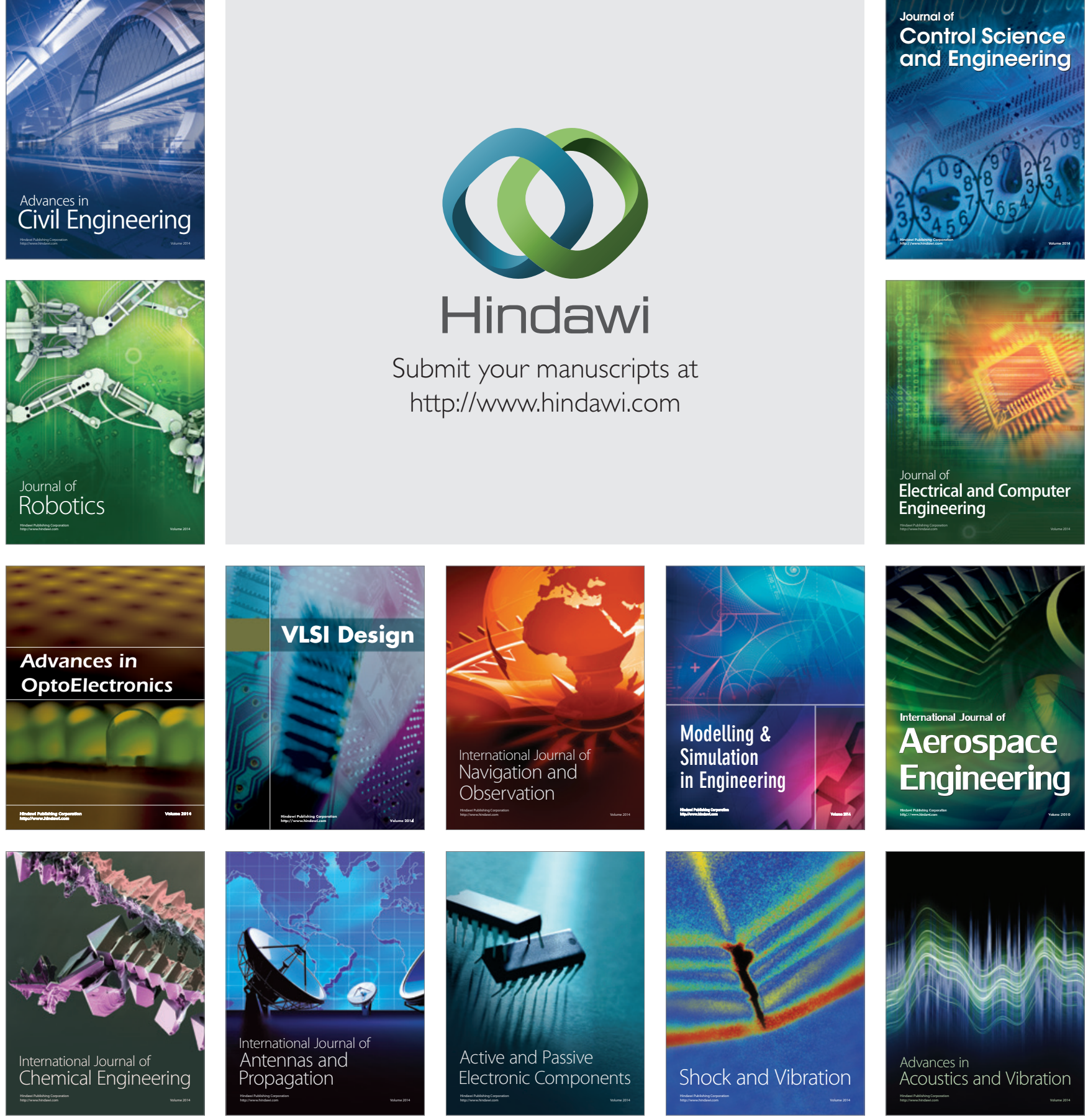